\title{
Global continuation of the eigenvalues of a perturbed linear operator
}

\author{
Pierluigi Benevieri $^{1,2}$. Alessandro Calamai ${ }^{3}$. \\ Massimo Furi ${ }^{4}$ - Maria Patrizia Pera ${ }^{4}$
}

Received: 19 July 2017 / Accepted: 1 November 2017 / Published online: 11 November 2017 (C) Fondazione Annali di Matematica Pura ed Applicata and Springer-Verlag GmbH Germany, part of Springer Nature 2017

\begin{abstract}
Let $E, F$ be real Banach spaces and $S$ the unit sphere of $E$. We study a nonlinear eigenvalue problem of the type $L x+\varepsilon N(x)=\lambda C x$, where $\varepsilon, \lambda$ are real parameters, $L: E \rightarrow$ $F$ is a Fredholm linear operator of index zero, $C: E \rightarrow F$ is a compact linear operator, and $N: S \rightarrow F$ is a compact map. Given a solution $(x, \varepsilon, \lambda) \in S \times \mathbb{R} \times \mathbb{R}$ of this problem, we say that the first element $x$ of the triple is a unit eigenvector corresponding to the eigenpair $(\varepsilon, \lambda)$. Assuming that $\lambda_{0} \in \mathbb{R}$ is such that the kernel of $L-\lambda_{0} C$ is odd dimensional and a natural transversality condition between the operators $L-\lambda_{0} C$ and $C$ is satisfied, we prove that, in the set of all the eigenpairs, the connected component containing $\left(0, \lambda_{0}\right)$ is either unbounded
\end{abstract}

Dedicated to the memory of our friend and outstanding mathematician Russell Johnson.

A. Calamai is partially supported by G.N.A.M.P.A. - INdAM (Italy). The first, second and fourth authors are members of the Gruppo Nazionale per l'Analisi Matematica, la Probabilità e le loro Applicazioni (GNAMPA) of the Istituto Nazionale di Alta Matematica (INdAM).

$凶$ Alessandro Calamai

calamai@dipmat.univpm.it

Pierluigi Benevieri

pierluigi.benevieri@unifi.it; pluigi@ime.usp.br

Massimo Furi

massimo.furi@math.unifi.it

Maria Patrizia Pera

mpatrizia.pera@unifi.it

1 Dipartimento di Matematica e Informatica, Università degli Studi di Firenze, Via S. Marta 3, 50139 Florence, Italy

2 Instituto de Matemática e Estatística, Universidade de São Paulo, Rua do Matão 1010, São Paulo, SP CEP 05508-090, Brasil

3 Dipartimento di Ingegneria Civile, Edile e Architettura, Università Politecnica delle Marche, Via Brecce Bianche, 60131 Ancona, Italy

4 Dipartimento di Matematica e Informatica "Ulisse Dini”, Università degli Studi di Firenze, Via S. Marta 3, 50139 Florence, Italy 
or meets an eigenpair $\left(0, \lambda_{1}\right)$, with $\lambda_{1} \neq \lambda_{0}$. Our approach is topological and based on the classical Leray-Schauder degree.

Keywords Fredholm operators · Nonlinear spectral theory · Eigenvalues · Eigenvectors · Degree theory

Mathematics Subject Classification 47J10 · 47A75 · 47H11

\section{Introduction}

Let $L$ be a self-adjoint operator defined on a real Hilbert space $H$, and let $N: H \rightarrow H$ be a (nonlinear) continuous map. Consider the following so-called nonlinear eigenvalue problem, depending on the real parameters $\varepsilon, \lambda$ :

$$
\left\{\begin{array}{l}
L x+\varepsilon N(x)=\lambda x, \\
\|x\|=1 .
\end{array}\right.
$$

Under the assumptions that $\lambda_{0} \in \mathbb{R}$ is an isolated simple eigenvalue of $L$ and that $N$ is Lipschitz continuous, Chiappinelli in [7] investigated a "persistence" property of the eigenvalues and eigenvectors of problem (1.1) for small values of $\varepsilon$. More precisely, he proved that, defined in a neighborhood $V$ of $0 \in \mathbb{R}$, there exist two $H$-valued Lipschitz curves, $\varepsilon \mapsto x_{\varepsilon}^{1}$ and $\varepsilon \mapsto x_{\varepsilon}^{2}$, as well as two real Lipschitz functions, $\varepsilon \mapsto \lambda_{\varepsilon}^{1}$ and $\varepsilon \mapsto \lambda_{\varepsilon}^{2}$, such that for $i=1,2$ and $\varepsilon \in V$ one has

$$
L x_{\varepsilon}^{i}+\varepsilon N\left(x_{\varepsilon}^{i}\right)=\lambda_{\varepsilon}^{i} x_{\varepsilon}^{i}, \quad\left\|x_{\varepsilon}^{i}\right\|=1 .
$$

In particular, when $\varepsilon=0$, these four functions satisfy $x_{0}^{i}=x^{i}, \lambda_{0}^{i}=\lambda_{0}$, where $x^{1}$ and $x^{2}$ are the two unit eigenvectors of $L$ corresponding to the simple eigenvalue $\lambda_{0}$.

After the result of Chiappinelli, in a series of papers [6,8-11] the above property of "local" persistence of the eigenvalues and eigenvectors was extended to the case in which the multiplicity of the eigenvalue $\lambda_{0}$ is bigger than one. In this case the set of unit eigenvectors of $L$ corresponding to $\lambda_{0}$ is the $(n-1)$-dimensional unit sphere $S^{n-1}=S \cap \operatorname{Ker}\left(L-\lambda_{0} I\right)$, where $S$ is the unit sphere in $H, I$ is the identity of $H$ and $n$ is the multiplicity of $\lambda_{0}$.

Actually, the results of the recent papers $[6,11]$ are obtained in the more general context of real Banach spaces. To better explain these results, consider the system

$$
\left\{\begin{array}{l}
L x+\varepsilon N(x)=\lambda C x \\
x \in \partial \Omega
\end{array}\right.
$$

where $L, C: E \rightarrow F$ are bounded linear operators between real Banach spaces, $\Omega$ is an open subset of $E$ containing the origin, and $N: \bar{\Omega} \rightarrow F$ is a continuous map.

A solution of (1.2) is a triple $(x, \varepsilon, \lambda)$ which satisfies the system. The first element of the triple is a $\partial \Omega$-eigenvector (or a unit eigenvector, when $\Omega$ is the unit ball of $E$ ) of problem (1.2) corresponding to the eigenpair $(\varepsilon, \lambda)$. The solutions and the eigenpairs with $\varepsilon=0$ are said to be trivial.

In [6] we studied the system (1.2), assuming that $N$ is a locally $\alpha$-Lipschitz map, where $\alpha$ stands for the Kuratowski measure of noncompactness [16] (see also [2]). Observe that this condition includes the case in which $N$ is the sum of a locally Lipschitz (e.g., $C^{1}$ ) map and a compact nonlinear operator.

In the main result of [6] we proved that if $\lambda_{0} \in \mathbb{R}$ is such that 
- the operator $L-\lambda_{0} C$ is Fredholm of index zero,

- the set $\Sigma=\partial \Omega \cap \operatorname{Ker}\left(L-\lambda_{0} C\right)$ of the trivial $\partial \Omega$-eigenvectors is nonempty and compact,

- $\operatorname{Ker}\left(L-\lambda_{0} C\right)$ is odd dimensional,

- the transversality condition $\left(L-\lambda_{0} C\right)(E)+C\left(\operatorname{Ker}\left(L-\lambda_{0} C\right)\right)=F$ holds,

then we get the persistence of at least one element of $\Sigma$.

All the cited results have a "local" nature since, as pointed out, they are valid only for small values of the parameter $\varepsilon$. In this paper, instead, we investigate the problem regarding the "global" continuation of eigenvalues and eigenvectors. For this purpose, we restrict the setting of [6], assuming, in addition, that the operators $N$ and $C$ are compact, and $\Omega$ is bounded. Obviously, because of the compactness of $C$, the first one of the above four hypotheses is the same as requiring that $L$ is Fredholm of index zero. The second one is automatically satisfied, $\Omega$ being bounded. Under these assumptions, our main result, Theorem 3.7, states the following "global continuation" property of the eigenpairs:

In the set of all the eigenpairs of (1.2), the connected component containing $\left(0, \lambda_{0}\right)$ is either unbounded or meets a trivial eigenpair $\left(0, \lambda_{1}\right)$ with $\lambda_{1} \neq \lambda_{0}$.

Going back to Chiappinelli's problem, we observe that, what in (1.2) is the operator $C$, in (1.1) is the identity $I$ of a Hilbert space $H$. Therefore, if $H$ is infinite dimensional, $I$ is not compact, and one may think that our global existence result does not apply in this case. However, it does apply when some appropriate assumptions on $L$ are satisfied. For example, when $L$ is a compact (not necessarily self-adjoint) operator. In fact, in this case, putting $\lambda=1 / \mu$ and $\varepsilon=-\sigma / \mu$, the equation $L x+\varepsilon N(x)=\lambda x$ becomes $x+\sigma N(x)=\mu L x$, which is of the form (1.2). Obviously, our result applies also when $L$ is of the type $\lambda_{*} I-C$, with $\lambda_{*} \in \mathbb{R}$ and $C$ a compact operator.

Our arguments are essentially topological and mainly based upon degree theory. Let us point out that here we use the classical Leray-Schauder degree, while the results in [6] were obtained applying a different degree theory, which was developed in $[3,4]$ (see also [5,18]) for a class of noncompact perturbations of Fredholm maps of index zero between Banach spaces, called $\alpha$-Fredholm maps, whose definition is related to the measure of noncompactness $\alpha$.

In the last section of the paper, we provide some examples, more or less complicated, illustrating our main result.

We leave as an open problem the validity of a corresponding "global continuation" property of the solution triples (see Conjecture 3.9). Although such a conjecture is supported by some examples, up to now we have not been able either to prove or disprove it.

\section{Preliminaries}

We recall that a subset $X$ of a metric space is locally compact if any point of $X$ admits a compact neighborhood in $X$. Obviously any compact set, as well as any relatively open subset of a locally compact set, is locally compact. The union of two locally compact sets needs not be locally compact (think about an open disk in $\mathbb{C}$ and add a point to the boundary).

Let $f: X \rightarrow Y$ be a continuous map between metric spaces. We recall that $f$ is said to be proper if $f^{-1}(K)$ is compact for any compact subset $K$ of $Y$. It is easy to check that, in this case, $f$ sends closed sets into closed sets. The map $f$ is said to be compact if $f(A)$ is relatively compact whenever $A \subseteq X$ is bounded.

Let $E$ be a real Banach space. A compact vector field on a subset $X$ of $E$ is a map of the type $f=I-g$, where $I$ denotes the identity of $E$ and $g: X \rightarrow E$ is a compact map. One can check that, in this case, $f$ is proper on any bounded and closed subset of $X$. 
Let $\Omega$ be a (possibly empty) bounded and open subset of $E$. Denote by $\bar{\Omega}$ and $\partial \Omega$ the closure and the boundary of $\Omega$, respectively. Call admissible any pair $(f, \Omega)$, where $f: X \rightarrow E$ is a compact vector field such that $\bar{\Omega} \subseteq X$ and $0 \notin f(\partial \Omega)$. A map $H: \bar{\Omega} \times[0,1] \rightarrow E$ of the type $(x, t) \mapsto x-G(x, t)$ is said to be an admissible homotopy if the image of $G$ is relatively compact and $(H(\cdot, t), \Omega)$ is an admissible pair for any $t \in[0,1]$.

In a seminal paper [15], Jean Leray and Juliusz Pawel Schauder extended the Brouwer topological degree to the infinite dimensional context by proving the existence of a function that to any admissible pair assigns an integer, denoted by $\operatorname{deg}_{L S}(f, \Omega)$ and called the LeraySchauder degree of $f$ in $\Omega$ (with target $0 \in E$ ), satisfying the following fundamental properties:

- (Normalization) Let $\Omega$ be a bounded open subset of $E$ and let $I$ be the identity of $E$. If $0 \in \Omega$, then

$$
\operatorname{deg}_{L S}(I, \Omega)=1 .
$$

- (Additivity) Let $(f, \Omega)$ be an admissible pair, and $\Omega_{1}, \Omega_{2}$ two disjoint open subsets of $\Omega$ such that $f^{-1}(0) \cap \Omega \subseteq \Omega_{1} \cup \Omega_{2}$. Then,

$$
\operatorname{deg}_{L S}(f, \Omega)=\operatorname{deg}_{L S}\left(f, \Omega_{1}\right)+\operatorname{deg}_{L S}\left(f, \Omega_{2}\right) .
$$

- (Homotopy invariance) If $H: \bar{\Omega} \times[0,1] \rightarrow E$ is an admissible homotopy, then $\operatorname{deg}_{L S}(H(\cdot, t), \Omega)$ does not depend on $t \in[0,1]$.

Other properties can be deduced from the above fundamental ones (see [1] for an axiomatic approach to degree theory). We only mention three of them.

- (Excision) Let $(f, \Omega)$ be an admissible pair, and $\Omega_{0}$ an open subset of $\Omega$ such that $f^{-1}(0) \cap \Omega \subseteq \Omega_{0}$. Then,

$$
\operatorname{deg}_{L S}(f, \Omega)=\operatorname{deg}_{L S}\left(f, \Omega_{0}\right) .
$$

- (Boundary dependence) Let $\left(f_{1}, \Omega\right)$ and $\left(f_{2}, \Omega\right)$ be two admissible pairs. If $f_{1}(x)=$ $f_{2}(x)$ for all $x \in \partial \Omega$, then

$$
\operatorname{deg}_{L S}\left(f_{1}, \Omega\right)=\operatorname{deg}_{L S}\left(f_{2}, \Omega\right) .
$$

- (Existence) Let $(f, \Omega)$ be an admissible pair. If

$$
\operatorname{deg}_{L S}(f, \Omega) \neq 0,
$$

then the equation $f(x)=0$ has at least one solution in $\Omega$.

In some sense, $\operatorname{deg}_{L S}(f, \Omega)$ is an algebraic count of the solutions in $\Omega$ of the equation $f(x)=0$.

Let $f$ be a linear compact vector field on $E$. That is, $f=I-A$, where $A$ is a compact linear operator acting on $E$. Clearly, given a bounded open neighborhood $\Omega$ of the origin $0 \in E$ and $\mu \in \mathbb{R}$, the pair $(I-\mu A, \Omega)$ is admissible if and only if $\mu$ is not a characteristic value of $A$; that is, if and only if $\lambda=1 / \mu$ is not an eigenvalue of $A$. In [15] it was shown that if $(I-\mu A, \Omega)$ is admissible, then $\operatorname{deg}_{L S}(I-\mu A, \Omega)$ is either 1 or -1 . Moreover, if $\mu_{0}$ is a characteristic value of $A$, then the function $\mu \mapsto \operatorname{deg}_{L S}(I-\mu A, \Omega)$, defined for $1 / \mu$ not in the spectrum $\sigma(A)$ of $A$, has a sign-jump crossing $\mu_{0}$ if and only if the algebraic multiplicity of $\mu_{0}$ is odd. Recall that the algebraic multiplicity of $\mu_{0}$ is the dimension of $\cup_{n=1}^{\infty} \operatorname{Ker}\left(I-\mu_{0} A\right)^{n}$, which is necessarily finite, due to the compactness of $A$, but greater than or equal to the geometric multiplicity of $\mu_{0}$, which is the dimension of $\operatorname{Ker}\left(I-\mu_{0} A\right)$. 
Clearly, the two multiplicities coincide if and only if $\operatorname{Ker}\left(I-\mu_{0} A\right)=\operatorname{Ker}\left(I-\mu_{0} A\right)^{2}$. It is known that the set of characteristic values of a compact operator is closed in $\mathbb{R}$, and any such value is isolated.

Let $E$ and $F$ be two real Banach spaces and let $L(E, F)$ denote the Banach space of all bounded linear operators from $E$ into $F$.

Let $L, C \in L(E, F)$ and consider the equation $L x-\lambda C x=0$ depending on a parameter $\lambda \in \mathbb{R}$. By abuse of terminology, one says that $\lambda_{0}$ is an eigenvalue of $L x-\lambda C x=0$ if this equation admits nontrivial solutions. Therefore, hereafter, if $A$ is an endomorphism of $E$, the two statements " $\lambda_{0}$ is an eigenvalue of the operator $A$ " and " $\lambda_{0}$ is an eigenvalue of the equation $A x=\lambda x$ " will be regarded as equivalent. Of course, for coherence, if $\mu_{0}$ is a characteristic value of $A$, we could equivalently say that $\mu_{0}$ is an eigenvalue of the equation $x-\mu A x=0$. Nevertheless, we will avoid using this terminology for the sake of clarity.

Recall that an operator $L \in L(E, F)$ is called Fredholm if both the spaces $\operatorname{Ker} L$ and coKer $L:=F / L(E)$ have finite dimension. Consequently, the image $L(E)$ of $L$ is closed in $F$. In fact, in the context of Banach spaces, the open mapping theorem implies that the image of a bounded linear operator is closed, whenever it admits a closed complement.

The index of a Fredholm operator $L$ is the integer

$$
\text { ind } L=\operatorname{dim} \operatorname{Ker} L-\operatorname{dim} \operatorname{coKer} L \text {. }
$$

One can easily check that any linear operator from $\mathbb{R}^{k}$ to $\mathbb{R}^{s}$ has index $k-s$.

A Fredholm operator of index $r \in \mathbb{Z}$ will be called a $\Phi_{r}$-operator, or a $\Phi$-operator if its index is not specified. The set of Fredholm operators of index $r$ from $E$ into $F$ will be denoted by $\Phi_{r}(E, F)$, while $\Phi(E, F)$ is the set of all Fredholm operators.

It is known that, given any $r \in \mathbb{Z}, \Phi_{r}(E, F)$ is an open subset of $L(E, F)$; therefore, so is the set $\Phi(E, F)$.

The following important properties of Fredholm operators will be utilized in Sect. 4:

- If $L \in \Phi_{r}(E, F)$ and $C \in L(E, F)$ is compact, then $L+C \in \Phi_{r}(E, F)$;

- The composition of two Fredholm operators is a Fredholm operator whose index is the sum of the indices of the composite operators.

\section{Results}

Let $E$ and $F$ be real Banach spaces. Consider the problem

$$
\left\{\begin{array}{l}
L x+\varepsilon N(x)=\lambda C x, \\
x \in \partial \Omega,
\end{array}\right.
$$

where $\varepsilon, \lambda \in \mathbb{R}$ and $L, C, N, \Omega$ satisfy the following basic assumptions:

- $L: E \rightarrow F$ is a linear Fredholm operator of index zero;

- $C: E \rightarrow F$ is a compact linear operator;

- $\Omega$ is a bounded open neighborhood of the origin of $E$;

- $N: \mathcal{D} \rightarrow F$ is a compact map whose domain $\mathcal{D} \subseteq E$ contains the boundary $\partial \Omega$ of $\Omega$.

A solution of (3.1) is a triple $(x, \varepsilon, \lambda)$ which satisfies the system. The first element $x \in \partial \Omega$ is a $\partial \Omega$-eigenvector (or a unit eigenvector, when $\Omega$ is the unit ball of $E$ ) of problem (3.1) corresponding to the eigenpair $(\varepsilon, \lambda)$.

The solutions and the eigenpairs with $\varepsilon=0$ are said to be trivial. Therefore, all the other solutions or eigenpairs are called nontrivial. 
Hereafter, the set of the solutions of (3.1) will be denoted by $\mathcal{S}$ and the set of the eigenpairs by $\mathcal{E}$. Notice that $\mathcal{E}$ is the projection of $\mathcal{S}$ into $\mathbb{R}^{2}$.

In the special case in which $\mathcal{D}=E$ and $N$ is linear, the choice of $\Omega$ is unimportant. Therefore, in this case, we will assume that $\Omega$ is the unit (open) ball of $E$, and problem (3.1) might be written as

$$
\left\{\begin{array}{l}
L x+\varepsilon N x=\lambda C x, \\
\|x\|=1
\end{array}\right.
$$

Anyhow, whether or not $N$ is linear, when $\Omega$ is the unit ball, the expression $x \in \partial \Omega$ could be replaced by $\|x\|=1$.

Our main result concerning problem (3.1) is Theorem 3.7, which asserts, in particular, that, if $\operatorname{Ker} L$ is odd dimensional and the transversality condition

$$
L(E)+C(\operatorname{Ker} L)=F
$$

is satisfied, then, in the set of all the eigenpairs, the connected component containing the "starting" eigenpair $(0,0)$ is either unbounded or meets a trivial eigenpair, different from the starting one.

In order to prove Theorem 3.7, we need some preliminary results.

The next remark will be useful in order to check that the transversality condition (3.3) holds for some examples that we will provide in Sect. 4. Recalling that the sum of a Fredholm operator $L$ and a compact operator $C$ is Fredholm of the same index as $L$, in some examples $L$ will be replaced by $L-\lambda_{0} C$, with $\lambda_{0} \in \mathbb{R}$. This will be convenient in checking the condition (3.7), whose (3.3) is a special case.

Remark 3.1 Let $L \in \Phi_{0}(E, F)$ and $C \in L(E, F)$. Then, (3.3) is equivalent to each one of the following conditions:

$$
\begin{aligned}
& L(E) \oplus C(\operatorname{Ker} L)=F, \\
& u \in \operatorname{Ker} L \text { and } C u \in L(E) \Longrightarrow u=0 .
\end{aligned}
$$

The assertion "(3.3) $\Longrightarrow$ (3.4)" is an easy consequence of the fact that Ker $L$ and coKer $L$ have the same dimension, while the converse implication is obvious.

To see that (3.4) and (3.5) are equivalent, observe that from (3.4) one gets that $\operatorname{dim} C(\operatorname{Ker} L)$ equals codim $L(E)$, which is the same as $\operatorname{dim} \operatorname{Ker} L$. Hence, $C$ is injective on $\operatorname{Ker} L$, and this implies (3.5), since $L(E) \cap C(\operatorname{Ker} L)=\{0\}$. The converse implication is also true because, if (3.5) holds, the restriction of $C$ to Ker $L$ is one-to-one and, consequently, $C(\operatorname{Ker} L)$ has the same dimension as $\operatorname{Ker} L$, which equals the codimension of $L(E)$. Thus, (3.4) follows, since (3.5) implies $L(E) \cap C(\operatorname{Ker} L)=\{0\}$.

Remark 3.2 Let, as above, $L \in \Phi_{0}(E, F)$ and $C \in L(E, F)$. Assume that $\operatorname{Ker} L$ is nontrivial and the transversality condition (3.3) is satisfied. Then $\lambda=0$ is an isolated eigenvalue of the equation $L x-\lambda C x=0$. That is, there exists $c>0$ such that $L-\lambda C$ is invertible if $0<|\lambda|<c$.

One way to check the above assertion is by writing $L-\lambda C$ in a block-matrix form according to the splittings

$$
E=E_{1} \oplus \operatorname{Ker} L, \quad F=L(E) \oplus C(\operatorname{Ker} L),
$$


where $E_{1}$ is any closed complement of $\operatorname{Ker} L$ and the decomposition of $F$ is according to condition (3.4) in Remark 3.1. We get

$$
L-\lambda C=\left(\begin{array}{cc}
L_{11}-\lambda C_{11} & 0 \\
-\lambda C_{21} & -\lambda C_{22}
\end{array}\right),
$$

which can be represented as the composition

$$
L-\lambda C=\left(\begin{array}{cc}
I_{11} & 0 \\
0 & -\lambda I_{22}
\end{array}\right)\left(\begin{array}{cc}
L_{11}-\lambda C_{11} & 0 \\
C_{21} & C_{22}
\end{array}\right),
$$

where, in the left matrix, $I_{11}$ and $I_{22}$ are the identities on $L(E)$ and $C(\operatorname{Ker} L)$, respectively. Since $L_{11}$ and $C_{22}$ are invertible, so is the right matrix for $|\lambda|$ sufficiently small. Hence, for the same values of $\lambda$, except $\lambda=0, L-\lambda C$ is invertible.

Remark 3.3 Let $L \in \Phi_{0}(E, F)$ and $C \in L(E, F)$. Assume that $\operatorname{Ker} L$ is nontrivial and the transversality condition (3.3) is satisfied. Given $\lambda_{*} \in \mathbb{R}$ such that $L-\lambda_{*} C$ is invertible, consider the endomorphism $\mathcal{L}=\left(L-\lambda_{*} C\right)^{-1} L$ of $E$. Then

$$
\operatorname{Ker} L=\operatorname{Ker} \mathcal{L}^{n}, \quad \forall n \in \mathbb{N} .
$$

To check the assertion, according to the splittings (3.6), one can write $\mathcal{L}$ in a block-matrix form as

$$
\begin{aligned}
\mathcal{L} & =\left[\left(\begin{array}{cc}
I_{11} & 0 \\
0 & -\lambda_{*} I_{22}
\end{array}\right)\left(\begin{array}{cc}
L_{11}-\lambda_{*} C_{11} & 0 \\
C_{21} & C_{22}
\end{array}\right)\right]^{-1}\left(\begin{array}{cc}
L_{11} & 0 \\
0 & 0
\end{array}\right) \\
& =\left(\begin{array}{cc}
\left(L_{11}-\lambda_{*} C_{11}\right)^{-1} & 0 \\
H & C_{22}^{-1}
\end{array}\right)\left(\begin{array}{cc}
I_{11} & 0 \\
0 & -\frac{1}{\lambda_{*}} I_{22}
\end{array}\right)\left(\begin{array}{cc}
L_{11} & 0 \\
0 & 0
\end{array}\right) \\
& =\left(\begin{array}{cc}
\left(L_{11}-\lambda_{*} C_{11}\right)^{-1} L_{11} & 0 \\
H L_{11} & 0
\end{array}\right) .
\end{aligned}
$$

From this representation of $\mathcal{L}$ one can easily deduce that $\operatorname{Ker} \mathcal{L}=\operatorname{Ker} \mathcal{L}^{2}$. Thus, by induction, one gets $\operatorname{Ker} \mathcal{L}=\operatorname{Ker} \mathcal{L}^{n}$ for all $n \in \mathbb{N}$. The above assertion now follows from the equality $\operatorname{Ker} L=\operatorname{Ker} \mathcal{L}$, which is obvious.

In order to prove Theorem 3.7, three preliminary lemmas are needed. The first one is related to the following transversality condition at an eigenvalue $\lambda_{0} \in \mathbb{R}$ of the equation $L x-\lambda C x=0$, of which (3.3) is the special case with $\lambda_{0}=0$ :

$$
\left(L-\lambda_{0} C\right)(E)+C\left(\operatorname{Ker}\left(L-\lambda_{0} C\right)\right)=F .
$$

Lemma 3.4 (On algebraic multiplicity) Let $L \in \Phi_{0}(E, F)$ and let $C \in L(E, F)$ be a compact operator. If $\lambda_{0} \in \mathbb{R}$ is an eigenvalue of the equation $L x-\lambda C x=0$ and the transversality condition (3.7) is satisfied, then $L-\lambda C$ is invertible, provided that $\left|\lambda-\lambda_{0}\right|$ is nonzero and sufficiently small. Moreover, if $\lambda_{*} \in \mathbb{R}$ is such that $L-\lambda_{*} C$ is invertible, then $\lambda_{0}-\lambda_{*}$ is a characteristic value of the compact endomorphism $\left(L-\lambda_{*} C\right)^{-1} C$ of $E$, whose algebraic multiplicity equals $\operatorname{dim} \operatorname{Ker}\left(L-\lambda_{0} C\right)$.

Proof Notice that (3.7) may be regarded as the transversality condition (3.3) for the equation $M x-\sigma C x=0$, where $M=L-\lambda_{0} C$ and $\sigma=\lambda-\lambda_{0}$. Therefore, taking into account Remark 3.3, one gets

$$
\operatorname{Ker} M=\operatorname{Ker} \mathcal{M}^{n}, \quad \forall n \in \mathbb{N},
$$


where $\mathcal{M}=\left(M-\sigma_{*} C\right)^{-1} M$ and $\sigma_{*}=\lambda_{*}-\lambda_{0}$. Thus, from the equality

$$
\mathcal{M}=I+\sigma_{*}\left(M-\sigma_{*} C\right)^{-1} C,
$$

that can be easily verified by composing both its sides with the invertible operator $M-\sigma_{*} C \in$ $L(E, F)$, one gets that $-\sigma_{*}=\lambda_{0}-\lambda_{*}$ is a characteristic value of $\left(M-\sigma_{*} C\right)^{-1} C$. Since this operator is equal to $\left(L-\lambda_{*} C\right)^{-1} C$, one has

$$
\mathcal{M}=I+\left(\lambda_{*}-\lambda_{0}\right)\left(L-\lambda_{*} C\right)^{-1} C .
$$

The assertion now follows from (3.8) and the definition of the algebraic multiplicity of a characteristic value of a compact endomorphism.

Lemma 3.5 appears very natural and intuitive. Its proof, however, seems to us not to be trivial and we are unable to provide a simpler one.

Lemma 3.5 (On circumnavigation) Let $K$ be a compact subset of a plane $\mathcal{P}$ and $U$ an open neighborhood of $K$ in $\mathcal{P}$. Assume that a straight line $\mathcal{L}$ in $\mathcal{P}$ meets $K$ just at one point $p$. If two points in $\mathcal{L} \backslash\{p\}$ are opposite and sufficiently close to $p$, then in $U \backslash K$ there exists a closed path, running through them, whose winding number around $p$ is nonzero. In particular, the points belong to the same connected component of $U \backslash K$.

Proof We may assume that $K$ is a subset of $\mathbb{R}^{2}$ (with the usual $x$ and $y$ coordinates), that $p$ is the origin of $\mathbb{R}^{2}$, that the line $\mathcal{L}$ is the $y$-axis, and that the neighborhood $U$ is bounded.

Let us first show that there exists a $C^{\infty}$ function $\varphi: \mathbb{R}^{2} \rightarrow \mathbb{R}$ with the following properties:

(1) $\frac{\partial \varphi}{\partial y}(0, y) y>0$ for all $y \neq 0$;

(2) $\max \{\varphi(x, y):(x, y) \in K\}<\min \{\varphi(x, y):(x, y) \in \partial U\}$.

To see this, we seek $\varphi$ among the functions of the type

$$
(x, y) \mapsto k \psi(x, y)+\omega(x / a) y^{2},
$$

where $a$ and $k$ are positive constants, $\psi$ is a nonnegative smooth function which vanishes exactly on the closed set $K \cup \mathcal{L}$, and $\omega: \mathbb{R} \rightarrow[0,1]$ is smooth, zero outside the interval $(-2,2)$ and such that $\omega(x)=1$ for $|x| \leq 1$. The existence of the functions $\psi$ and $\omega$ is a well known fact in real analysis and differential geometry.

Notice that $k \psi(0, y)+\omega(0 / a) y^{2} \equiv y^{2}$ for all $a$ and $k$. Therefore, property (1) is satisfied by any function of the above class.

We need to find $a$ and $k$ in order to satisfy (2). For this purpose, we first choose $a$ in such a way that $\alpha(a)<\beta(a)$, where

$$
\alpha(a)=\max \left\{y^{2}:(x, y) \in K,|x| \leq 2 a\right\}
$$

and

$$
\beta(a)=\min \left\{y^{2}:(x, y) \in \partial U,|x| \leq 2 a\right\} .
$$

This is possible because of the compactness of $K$ and $\partial U$, and the fact that $K \cap \mathcal{L}$ is the origin of $\mathbb{R}^{2}$, which is contained in $U$. Actually, it is easy to verify that the two functions $\alpha$ and $\beta$ are monotone and that, as $t \rightarrow 0^{+}$, one has $\alpha(t) \rightarrow 0$ and $\beta(t) \rightarrow \ell>0$, where $\ell=\min \left\{y^{2}:(0, y) \in \partial U\right\}$.

Thus, for any $k>0$ we have

$$
\max \left\{k \psi(x, y)+\omega(x / a) y^{2}:(x, y) \in K\right\} \leq \alpha(a)
$$


and

$$
\min \left\{k \psi(x, y)+\omega(x / a) y^{2}:(x, y) \in \partial U,|x| \leq a\right\} \geq \beta(a) .
$$

Consequently, if we take $k$ such that

$$
k \min \{\psi(x, y):(x, y) \in \partial U,|x| \geq a\} \geq \beta(a),
$$

the function

$$
\varphi(x, y):=k \psi(x, y)+\omega(x / a) y^{2}
$$

satisfies condition (2), as well. In fact, recalling that $a$ has been chosen such that $\alpha(a)<\beta(a)$, one has

$$
\max \{\varphi(x, y):(x, y) \in K\} \leq \alpha(a)
$$

and

$$
\beta(a) \leq \min \{\varphi(x, y):(x, y) \in \partial U\} .
$$

In what follows, a point in $\mathcal{L} \backslash\{p\}$ is sufficiently close to $p$ when it belongs to the open neighborhood $V=\{(x, y) \in U: \varphi(x, y)<\beta(a)\}$ of $K$.

Because of Sard's Lemma, there exists a value $c$, between the two numbers $\alpha(a)$ and $\beta(a)$, which is regular for the restriction of $\varphi$ to $U$. Consequently, $\varphi^{-1}(c) \cap U$ is a compact, onedimensional, boundaryless manifold. In fact, it is a finite union of pairwise disjoint smooth Jordan curves contained in $U \backslash K$ (see, e.g., [17]). Notice that, due to the property (1) of $\varphi$, on the $y$-axis there are exactly two points, $p_{+}$and $p_{-}$, belonging to $\varphi^{-1}(c)$, and these points belong to $V$, therefore they are "sufficiently close to $p$ ". One of them, say $p_{+}$, must be above the origin and the other one below. (Actually, due to the particular choice of $\varphi$, we have $p_{+}=(0, \sqrt{c})$ and $p_{-}=(0,-\sqrt{c})$.)

We claim that $p_{+}$and $p_{-}$belong to the same curve of $\varphi^{-1}(c)$. In fact, if the curve containing $p_{+}$did not contain $p_{-}$, its intersection number with the $y$-axis, due to the transversal intersection ensured by property (1) of $\varphi$, would be either 1 or -1 , depending on the orientations of the curve and the $y$-axis (see, e.g., [14]). This would contradict the fact that the intersection number of a Jordan curve with a straight line is zero, and our claim is proved.

Obviously the Jordan curve of $\varphi^{-1}(c)$ containing $p_{+}$and $p_{-}$lies in $V$. Moreover, it surrounds the point $p$, meaning that $p$ is contained in the bounded region enclosed by the curve.

To prove the assertion take, in $(\mathcal{L} \backslash\{p\}) \cap V$, two points, $q_{+}$and $q_{-}$, which are opposite with respect to $p$. Consider a parameterization $\gamma_{0}: S^{1} \rightarrow V \backslash K$ of the above Jordan curve containing $p_{+}$and $p_{-}$and modify $\gamma_{0}$ homotopically (with values in $V \backslash K$ ) in such a way that the final path $\gamma_{1}$ runs through $q_{+}$and $q_{-}$(this can be done, for example, by modifying portions of the path just by moving along the line $\mathcal{L}$ and remaining in $V \backslash\{p\})$. Now, recall that the winding number of a Jordan curve around a surrounded point is either 1 or -1 , according to the orientation. Thus, due to the homotopy invariance property of the winding number, the final path satisfies the assertion.

Lemma 3.6 is a Whyburn-type topological result and is crucial to the proof of Theorem 3.7.

Lemma 3.6 ([13]) Let $Y_{0}$ be a compact subset of a locally compact metric space $Y$. Assume that every compact subset of $Y$ containing $Y_{0}$ has nonempty boundary. Then $Y \backslash Y_{0}$ contains a connected set whose closure in $Y$ is noncompact and intersects $Y_{0}$. 
We are now ready to state and prove our main result regarding problem (3.1).

Theorem 3.7 (Global continuation of eigenpairs) Consider the problem

$$
\left\{\begin{array}{l}
L x+\varepsilon N(x)=\lambda C x, \\
x \in \partial \Omega,
\end{array}\right.
$$

where $L: E \rightarrow F$ is a Fredholm operator of index zero between real Banach spaces, $C: E \rightarrow$ $F$ is a compact linear operator, $\Omega$ is a bounded open neighborhood of the origin of $E$, $N: \mathcal{D} \rightarrow F$ is a compact map whose domain $\mathcal{D}$ contains the boundary $\partial \Omega$ of $\Omega, \varepsilon$ and $\lambda$ are real parameters.

Assume that, for some $\lambda_{0} \in \mathbb{R}, \operatorname{Ker}\left(L-\lambda_{0} C\right)$ is odd dimensional and the transversality condition

$$
\left(L-\lambda_{0} C\right)(E)+C\left(\operatorname{Ker}\left(L-\lambda_{0} C\right)\right)=F
$$

is satisfied. Then, in the set of all the eigenpairs $(\varepsilon, \lambda)$ of the problem, the connected component containing $\left(0, \lambda_{0}\right)$ is either unbounded or meets a trivial eigenpair $\left(0, \lambda_{1}\right)$ with $\lambda_{1} \neq \lambda_{0}$.

Proof We will prove first that the set $\mathcal{E}$ of the eigenpairs of (3.1) is closed.

Then, we will show that there exists a function $\delta: \mathbb{R}^{2} \rightarrow \mathbb{Z}$ which is locally constant on the open set $\mathbb{R}^{2} \backslash \mathcal{E}$ and has the property that $\lambda \mapsto \delta(0, \lambda)$ has a sign-jump crossing $\lambda_{0}$.

Finally, we will apply Lemma 3.6 to a suitable pair $\left(Y, Y_{0}\right)$ of subsets of $\mathcal{E}$, whose assertion, if denied, would contradict Lemma 3.5.

Without loss of generality, we may assume that $N$ is defined (and compact) on the whole space $E$. In fact, because of the Dugundji Extension Theorem (see [12]), the restriction of $N$ to $\partial \Omega$ admits a continuous extension to $E$ whose values are contained in the convex closure of $N(\partial \Omega)$, which is a compact set.

Define $\Psi: E \times \mathbb{R}^{2} \rightarrow F$ by

$$
\Psi(x, \varepsilon, \lambda)=L x+\varepsilon N(x)-\lambda C x .
$$

This map is proper on bounded closed sets, as a compact perturbation of the operator $(x, \varepsilon, \lambda) \mapsto L x$ which, being Fredholm (of index 2), is proper on bounded closed subsets of $E \times \mathbb{R}^{2}$. Let

$$
\mathcal{S}=\left\{(x, \varepsilon, \lambda) \in \partial \Omega \times \mathbb{R}^{2}: \Psi(x, \varepsilon, \lambda)=0\right\}=\Psi^{-1}(0) \cap\left(\partial \Omega \times \mathbb{R}^{2}\right)
$$

be the closed subset of $E \times \mathbb{R}^{2}$ of the solution triples of (3.1). Due to the above property of the map $\Psi$, any bounded subset of $\mathcal{S}$ is relatively compact. This implies that the set $\mathcal{E}$ of the eigenpairs of (3.1), which is the projection of $\mathcal{S}$ into $\mathbb{R}^{2}$, is closed. In particular, it is locally compact.

As pointed out in Remark 3.2, the transversality condition (3.7) ensures the existence of $\lambda_{*} \in \mathbb{R}$ such that the operator $L-\lambda_{*} C$ is invertible. Composing $\Psi$ with $\left(L-\lambda_{*} C\right)^{-1}$, we obtain a map $\Phi: E \times \mathbb{R}^{2} \rightarrow E$ given by

$$
\Phi(x, \varepsilon, \lambda)=x+\varepsilon\left(L-\lambda_{*} C\right)^{-1} N(x)-\left(\lambda-\lambda_{*}\right)\left(L-\lambda_{*} C\right)^{-1} C x .
$$

Problem (3.1) is clearly equivalent to

$$
\left\{\begin{array}{l}
\Phi(x, \varepsilon, \lambda)=0, \\
x \in \partial \Omega,
\end{array}\right.
$$

and, consequently, $\Phi(x, \varepsilon, \lambda) \neq 0$ for any $(x, \mathcal{\varepsilon}, \lambda) \in \partial \Omega \times\left(\mathbb{R}^{2} \backslash \mathcal{E}\right)$. 
Now, observe that, given any $(\varepsilon, \lambda) \in \mathbb{R}^{2}$, the map $\Phi(\cdot, \varepsilon, \lambda)$ is a compact vector field on $E$ and, given $(\varepsilon, \lambda) \in \mathbb{R}^{2}$, the Leray-Schauder degree of $\Phi(\cdot, \varepsilon, \lambda)$ in $\Omega$ is defined whenever $(\varepsilon, \lambda) \notin \mathcal{E}$. Thus, we may define $\delta: \mathbb{R}^{2} \rightarrow \mathbb{Z}$ by

$$
\delta(\varepsilon, \lambda)=\left\{\begin{array}{l}
\operatorname{deg}_{L S}(\Phi(\cdot, \varepsilon, \lambda), \Omega) \quad \text { if } \quad(\varepsilon, \lambda) \in \mathbb{R}^{2} \backslash \mathcal{E}, \\
0 \text { if }(\varepsilon, \lambda) \in \mathcal{E} .
\end{array}\right.
$$

Notice that, because of the boundary dependence property of the degree, the function $\delta$ depends only on the restriction of $N$ to $\partial \Omega$. Moreover, due to the homotopy invariance property, $\delta$ is constant in the components of the set $\mathbb{R}^{2} \backslash \mathcal{E}$, which, being open in $\mathbb{R}^{2}$, is locally path connected. In particular, taking into account that $0 \in \Omega$, given any $\lambda \in \mathbb{R}$, the linearity of the compact vector field

$$
\Phi(\cdot, 0, \lambda)=I-\left(\lambda-\lambda_{*}\right)\left(L-\lambda_{*} C\right)^{-1} C
$$

implies that, in the components of $\mathbb{R}^{2} \backslash \mathcal{E}$ containing points with $\varepsilon=0$, the constant is either 1 or -1 (see [15]).

According to Lemma 3.4, the eigenvalue $\lambda_{0}$ of the equation $L x-\lambda C x=0$ is isolated. Thus, there exists $c>0$ such that $\delta(0, \lambda)= \pm 1$ for $0<\left|\lambda-\lambda_{0}\right|<c$.

We claim that the odd dimensionality of $\operatorname{Ker}\left(L-\lambda_{0} C\right)$ implies that $\lambda \mapsto \delta(0, \lambda)$ has a sign-jump crossing $\lambda_{0}$. To see this, observe first that, if $(0, \lambda) \in \mathbb{R}^{2} \backslash \mathcal{E}$, then

$$
\delta(0, \lambda)=\operatorname{deg}_{L S}\left(I-\left(\lambda-\lambda_{*}\right) A, \Omega\right),
$$

where $A$ is the compact operator $\left(L-\lambda_{*} C\right)^{-1} C$. Now, according to Lemma $3.4, \mu_{0}=$ $\lambda_{0}-\lambda_{*}$ is a characteristic value of $A$, whose algebraic multiplicity equals the dimension of $\operatorname{Ker}\left(L-\lambda_{0} C\right)$ which, by assumption, is odd. Thus, as pointed out in Sect. 2, our claim is a consequence of a well known property of the degree of a linear compact vector field.

Let $\mathcal{E}_{0}=\{(\varepsilon, \lambda) \in \mathcal{E}: \varepsilon=0\}$ denote the set of the trivial eigenpairs, and let $\mathcal{E}_{*}=\mathcal{E} \backslash \mathcal{E}_{0}$ be the set of the nontrivial ones. Since $A=\left(L-\lambda_{*} C\right)^{-1} C$ is a compact linear operator, it admits a countable number of isolated characteristic values $\mu=\lambda-\lambda_{*}$. Consequently, the topology of $\mathcal{E}_{0}$ is the discrete one. Therefore, since $\mathcal{E}_{0}$ is closed in $\mathcal{E}$, so is any subset of $\mathcal{E}_{0}$.

Denote by $Y_{0}$ the subset of $\mathcal{E}_{0}$ corresponding to the characteristic value $\mu_{0}=\lambda_{0}-\lambda_{*}$ of $A$ (i.e., $Y_{0}$ is the singleton $\left.\left\{\left(0, \lambda_{0}\right)\right\}\right)$ and consider the disjoint union

$$
Y=\mathcal{E}_{*} \cup Y_{0} .
$$

We want to apply Lemma 3.6 to the metric pair $\left(Y, Y_{0}\right)$. To accomplish this goal we need to show, first, that $Y$ is locally compact. Of course $Y$ is union of two locally compact sets: $\mathcal{E}_{*}$, which is relatively open in $\mathcal{E}$, and $Y_{0}$, which is compact. However, as pointed out before, the union of two locally compact spaces needs not be locally compact. Nevertheless, the required property of $Y$ can be shown by observing that $Y$ is equal to $\mathcal{E} \backslash\left(\mathcal{E}_{0} \backslash Y_{0}\right)$ and, consequently, it is a relatively open subset of the locally compact space $\mathcal{E}$ (recall that any subset of $\mathcal{E}_{0}$ is closed in $\mathcal{E}$ ).

Considering Lemma 3.6, we now show that the set $\mathcal{E}_{*}=Y \backslash Y_{0}$ of the nontrivial eigenpairs of (3.1) contains a connected set whose closure in $Y$ is noncompact and intersects $Y_{0}$.

Assume that this is false. Then, according to Lemma 3.6, in $Y$ there exists a compact set $K \supseteq Y_{0}$ such that $\partial K=\emptyset$. In particular, $K$ is relatively open in $Y$ and, therefore, there exists an open set $U$ of $\mathbb{R}^{2}$ such that $U \cap Y=K$. Due to the fact that $\mathcal{E} \backslash Y=\mathcal{E}_{0} \backslash Y_{0}$ is a closed subset of $\mathbb{R}^{2}$ which does not intersect $Y$, we may assume (taking $U$ smaller, if necessary) that $U$ is disjoint from $\mathcal{E} \backslash Y$, so that $U \cap \mathcal{E}=K$. Consequently, $U \backslash K$ does not intersect $\mathcal{E}$ and, as pointed out before, in this open set the integer valued function $\delta$ is locally constant. 
Now, according to Lemma 3.5 , if $\left(0, \lambda_{+}\right)$and $\left(0, \lambda_{-}\right)$are two points opposite $\left(0, \lambda_{0}\right)$ and sufficiently close to it, then they belong to the same connected component of $U \backslash K$. Thus $\delta\left(0, \lambda_{+}\right)=\delta\left(0, \lambda_{-}\right)$, contradicting the fact that the function $\lambda \mapsto \delta(0, \lambda)$ has a sign-jump at $\lambda_{0}$. The contradiction, because of Lemma 3.6, implies the existence of a connected subset $S$ of $\mathcal{E}_{*}=Y \backslash Y_{0}$ whose closure in $Y$ is noncompact and intersects $Y_{0}$. Now, denote by $\widehat{S}$ the connected component, in $\mathcal{E}$, containing $Y_{0}=\left\{\left(0, \lambda_{0}\right)\right\}$. Clearly $\widehat{S}$ contains the closure in $Y$ of $S$ (recall that the closure of a connected set is connected), as well as the closure $\bar{S}$ of $S$ in $\mathcal{E}$ (which is the same as the closure in $\mathbb{R}^{2}, \mathcal{E}$ being closed). If $\widehat{S}$ is unbounded, then our assertion holds true. If it is bounded, then, being closed in $\mathcal{E}$ (as well as in $\mathbb{R}^{2}$ ), it is a compact set. Therefore, it must contain at least one point $\left(0, \lambda_{1}\right)$ of $\mathcal{E} \backslash Y=\mathcal{E}_{0} \backslash Y_{0}$, since otherwise its closure in $Y$ would coincide with the closure in $\mathcal{E}$ and would be compact. Hence, also in this case, our assertion holds true.

Remark 3.8 Let, as above, $\mathcal{E}$ denote the set of all the eigenpairs of (3.1). From the proof of Theorem 3.7, and the existence property of the Leray-Schauder degree, one can easily deduce that if a given pair $\left(\varepsilon_{*}, \lambda_{*}\right)$ belongs to a connected component of $\mathbb{R}^{2} \backslash \mathcal{E}$ whose intersection with the $\lambda$-axis is nonempty, then the equation

$$
L x+\varepsilon_{*} N(x)-\lambda_{*} C x=0
$$

admits at least one solution in $\Omega$.

We close this section with a conjecture that, if true, would extend Theorem 3.7 in the special case when $\Omega$ is the unit ball.

Conjecture 3.9 (Global continuation of solution triples) Let L, C, N satisfy the basic assumptions of problem (3.1). Assume that $\Omega$ is the unit ball of $E$ and that $\lambda_{0} \in \mathbb{R}$ is such that $\operatorname{Ker}\left(L-\lambda_{0} C\right)$ is odd dimensional and the transversality condition

$$
\left(L-\lambda_{0} C\right)(E)+C\left(\operatorname{Ker}\left(L-\lambda_{0} C\right)\right)=F
$$

is satisfied. Then, there exists a unit eigenvector $x_{0} \in E$, corresponding to the trivial eigenpair $\left(0, \lambda_{0}\right)$, such that, in the set $\mathcal{S}$ of all the solutions of (3.1), the connected component containing $\left(x_{0}, 0, \lambda_{0}\right)$ is either unbounded or reaches a trivial solution $\left(x_{1}, 0, \lambda_{1}\right)$ with $\lambda_{1} \neq \lambda_{0}$.

\section{Examples}

Here we provide examples illustrating how Theorem 3.7 applies, as well as examples showing that the assumptions of this theorem, such as the transversality condition (3.7) and the odd dimensionality of the kernel of the unperturbed operator, cannot be omitted.

According to the notation of Theorem 3.7, in each example it will be clear what the spaces $E$ and $F$, the operators $L, N$ and $C$, the open subset $\Omega$ of $E$, and the starting eigenvalue $\lambda_{0}$ of the unperturbed equation $L x=\lambda C x$ are.

The following is, perhaps, the simplest example showing the existence of a bounded branch of eigenpairs connecting two different trivial elements.

Example 4.1 Let $E=F=\mathbb{R}^{2}$, with the standard $x$ and $y$ coordinates, and consider the linear problem

$$
\left\{\begin{array}{l}
x+\varepsilon y=\lambda x, \\
y+\varepsilon x=-\lambda y, \\
x^{2}+y^{2}=1 .
\end{array}\right.
$$


Here, $\Omega$ is the unit open disk of $\mathbb{R}^{2}$. Obviously, the operators are $L:(x, y) \mapsto(x, y)$, $N:(x, y) \mapsto(y, x)$ and $C:(x, y) \mapsto(x,-y)$. The unperturbed problem has two eigenvalues, $\lambda_{0}=1$ and $\lambda_{1}=-1$.

As one can easily check, the set $\mathcal{E}$ of the eigenpairs of (4.1) is the unit circle $\varepsilon^{2}+\lambda^{2}=1$. Therefore, the connected component of $\mathcal{E}$ containing the trivial element $\left(0, \lambda_{0}\right)=(0,1)$ is bounded and meets $(0,-1)$.

The eigenpairs $(\varepsilon, \lambda)$ can be represented, parametrically, as $(-\sin \theta, \cos \theta)$, with $\theta \in$ $[0,2 \pi]$ and, given $\theta$, the kernel of the linear operator

$$
L-(\sin \theta) N-(\cos \theta) C
$$

is spanned by the unit eigenvector

$$
u(\theta)=(x(\theta), y(\theta))=(\cos (\theta / 2), \sin (\theta / 2)) .
$$

Obviously, another continuous curve of unit eigenvectors of problem (4.1) is given by $\theta \mapsto-u(\theta), \theta \in[0,2 \pi]$. Both the curves $u$ and $-u$ are not closed. They can be "glued" to create a unique (continuous) closed curve whose image contains all the unit eigenvectors of (4.1). This curve, say $v:[0,4 \pi] \rightarrow \mathbb{R}^{2}$, is given by

$$
v(\theta)=(\cos (\theta / 2), \sin (\theta / 2)) .
$$

Notice that the kernel of $L-\lambda_{0} C$ is the $x$-axis, which is odd dimensional. The image under $C$ of this kernel is again the $x$-axis, which is orthogonal to the image of $L-\lambda_{0} C$. Thus, all the assumptions of Theorem 3.7 are satisfied.

Incidentally, we point out that, in this example, the assertion of Conjecture 3.9 is satisfied. In fact, for example, the connected set of solution triples

$$
\mathcal{C}=\{(u(\theta),-\sin \theta, \cos \theta): \theta \in[0, \pi]\}
$$

contains two trivial elements: the "starting one" $\left(u(0), 0, \lambda_{0}\right)=((1,0), 0,1)$, as well as the "reached one" $\left(u(\pi), 0, \lambda_{1}\right)=((0,-1), 0,-1)$. Obviously, in the set $\mathcal{S}$ of the solutions of (4.1), the connected component containing $\mathcal{C}$ has the same property.

In the following example, the unperturbed equation $L x=\lambda C x$ has a unique eigenvalue, $\lambda_{0}=0$. Since, as we shall see, all the assumption of Theorem 3.7 are satisfied, one concludes that there is an unbounded connected set of eigenpairs emanating from the trivial eigenpair $(0,0)$.

Example 4.2 Let $E$ denote the one-codimensional closed subspace of the Banach space $C^{1}([0,2 \pi], \mathbb{R})$ of the functions $x$ satisfying the condition $x(0)=x(2 \pi)$ and let $F=$ $C^{0}([0,2 \pi], \mathbb{R})$. Consider the problem

$$
\left\{\begin{array}{l}
x^{\prime}(t)+\varepsilon \sin t=\lambda x(t), \\
x(0)=x(2 \pi), \quad x \in \partial \Omega,
\end{array}\right.
$$

where $\Omega$ is the unit ball of the space $E$ endowed with the Banach norm

$$
\|x\|=\frac{1}{2}\left(\sup \{|x(t)|: t \in[0,2 \pi]\}+\sup \left\{\left|x^{\prime}(t)\right|: t \in[0,2 \pi]\right\}\right) .
$$

The above problem can be written in the form

$$
\left\{\begin{array}{l}
L x+\varepsilon N(x)=\lambda C x, \\
\|x\|=1,
\end{array}\right.
$$


where

- $L: E \rightarrow F$ is the bounded linear operator $x \mapsto x^{\prime}$;

- $N: E \rightarrow F$ is the (constant) map defined by $N(x)(t)=\sin t$;

- $C$ is the (compact) inclusion of $E$ into $F$.

Observe that the operator $L$ is Fredholm of index zero, because it is the composition of the inclusion $E \hookrightarrow C^{1}([0,2 \pi], \mathbb{R})$, which is Fredholm of index -1 , with the differential operator $x \in C^{1}([0,2 \pi], \mathbb{R}) \mapsto x^{\prime} \in F$, which is Fredholm of index 1 (being surjective with one-dimensional kernel).

Clearly, the unique eigenvalue of the unperturbed equation $L x=\lambda C x$ is $\lambda_{0}=0$. The kernel of $L$ is composed of the constant functions. Therefore, the transversality condition (3.7) is trivially satisfied, $C$ being the inclusion and the image of $L$ consisting of the functions in $C^{0}([0,2 \pi], \mathbb{R})$ with zero average.

Since the assumptions of Theorem 3.7 are satisfied and the unperturbed operator $L-\lambda C$ has only one eigenvalue, the set $\mathcal{E}$ of the eigenpairs of our problem is necessarily unbounded. More precisely, in the space $\mathcal{E}$, the component containing $(0,0)$ is unbounded. Actually, this is true not only when $N$ is the constant map defined above, but also when it is any compact map from $E$ into $F$. For example, the existence of an unbounded branch emanating from $(0,0)$ survives if in problem (4.2) we replace $\varepsilon \sin t$ with $\varepsilon g(t, x(t))$, where $g:[0,2 \pi] \times \mathbb{R} \rightarrow \mathbb{R}$ is any continuous function. In fact, the map $N: E \rightarrow F$ defined by $N(x)(t)=g(t, x(t))$ is compact, because it is the composition of the compact inclusion of $E$ into $F$ with a continuous self-map of $F$.

In our specific case (4.2), standard computations show that, given $(\varepsilon, \lambda)$, the problem

$$
\left\{\begin{array}{l}
x^{\prime}(t)+\varepsilon \sin t=\lambda x(t) \\
x(0)=x(2 \pi)
\end{array}\right.
$$

has a solution

$$
x(t)=\frac{\varepsilon}{1+\lambda^{2}}(\lambda \sin t+\cos t),
$$

which is unique if and only if $\lambda \neq 0$, and its norm is $|\varepsilon| / \sqrt{1+\lambda^{2}}$.

Thus, given a pair $(\varepsilon, 0)$, the function $\varepsilon \cos t$ is a particular solution. Consequently, when $\lambda=0$, problem (4.3) has infinitely many solutions: $x(t)=c+\varepsilon \cos t(c \in \mathbb{R})$, with $\|x\|=|c| / 2+|\varepsilon|$.

Therefore, recalling that $\partial \Omega$ is the unit sphere, the set $\mathcal{E}$ is the union of three connected sets. One is the horizontal set $[-1,1] \times\{0\}$, corresponding to the case $\lambda=0$. The other two are the left and right branches of the hyperbola

$$
\varepsilon^{2}-\lambda^{2}=1
$$

Thus, $\mathcal{E}$ is a connected set, since both the branches of the hyperbola have a point in common with the above horizontal set: $(-1,0)$ for the left branch and $(1,0)$ for the right one.

Observe that in this example, as in the previous one, the assertion of Conjecture 3.9 is satisfied. For example, the set $\mathcal{S}$ of solution triples of problem (4.2) contains the unbounded image of the (continuous) curve $\gamma:[-1,+\infty) \rightarrow \mathcal{S}$ starting from the trivial triple $(2,0,0)$ and given by

$$
\gamma(s)=\left\{\begin{array}{l}
(-2 s+(s+1) \cos (\cdot), s+1,0) \text { if }-1 \leq s \leq 0, \\
\left(\frac{1}{\sqrt{1+s^{2}}}(s \sin (\cdot)+\cos (\cdot)), \sqrt{1+s^{2}}, s\right) \text { if } s \geq 0
\end{array}\right.
$$


In the following example the unperturbed operator $L-\lambda C$ has infinitely many eigenvalues. Informally, two of them are connected by a bounded branch of eigenpairs, all the others are part of a unique unbounded branch.

Example 4.3 Let $E=F=\ell^{2}$ and denote by $\left\{e_{i}\right\}_{i \in \mathbb{N}}$ the standard basis of $\ell^{2}$. Here as $L$ we take the identity $I$ of $\ell^{2}$, which is obviously Fredholm of index zero. The compact (linear) operator $C$ is defined by $C e_{1}=e_{1}, C e_{2}=-e_{2}$ and $C e_{i}=\frac{1}{i} e_{i}$, for $i \geq 3$. The compact (nonlinear) map $N$ is given by $\left(\xi_{1}, \xi_{2}, \xi_{3}, \ldots\right) \mapsto\left(\xi_{2}, \xi_{1}, \frac{1}{3}, \frac{1}{4}, \frac{1}{5}, \cdots\right)$.

We consider the problem

$$
\left\{\begin{array}{l}
x+\varepsilon N(x)=\lambda C x \\
\|x\|=1
\end{array}\right.
$$

The transversality condition (3.7) is satisfied at any characteristic value of the compact operator $C$. Moreover, for any of these values, the kernel of $I-\lambda C$ is one dimensional.

One can see that (4.5) admits infinitely many trivial eigenpairs: $(0,-1),(0,1),(0,3)$, $(0,4),(0,5)$, and so on.

The set $\mathcal{E}$ of the eigenpairs has only two connected components. One is the circle with equation $\varepsilon^{2}+\lambda^{2}=1$. The other component is the union of the following infinite set of straight lines: $\lambda= \pm \varepsilon+3, \lambda= \pm \varepsilon+4, \lambda= \pm \varepsilon+5$, and so on.

The second set is connected, since any straight line intersects all the other ones with opposite angular coefficient. However, this connected set is the projection into $\mathbb{R}^{2}$ of infinitely many connected components in the set $\mathcal{S}$ of solution triples. Actually, any such a component is a straight line in $E \times \mathbb{R}^{2}$, which is "parallel" to $\mathbb{R}^{2}$ and satisfies the assertion of Conjecture 3.9. For example, the component over the line $\lambda=\varepsilon+3$ is the line $\left\{\left(e_{3}, \varepsilon, \lambda\right): \lambda=\varepsilon+3\right\}$, while the component over $\lambda=-\varepsilon+3$ is $\left\{\left(-e_{3}, \varepsilon, \lambda\right): \lambda=-\varepsilon+3\right\}$.

The component over the circle $\varepsilon^{2}+\lambda^{2}=1$ is more complicated to describe. However, it can be shown that it is a simple closed curve which satisfies the assertion of Conjecture 3.9. In fact, it contains the trivial solutions $\left(e_{1}, 0,1\right)$ and $\left(e_{2}, 0,-1\right)$.

In the following example, whose physical meaning is evident, $\lambda_{0}=-1$ is an eigenvalue of the unperturbed equation $L x-\lambda C x=0$. Although the transversality condition (3.7) is satisfied, the trivial eigenpair $(0,-1)$ is isolated in the set $\mathcal{E}$ of all the eigenpairs. Thus, assuming that Theorem 3.7 holds true, the absence of a branch starting from $(0,-1)$ implies that the kernel of $L-\lambda_{0} C$ must be even. As it is.

Example 4.4 Consider the differential equation

$$
\left\{\begin{array}{l}
x^{\prime \prime}(t)+\varepsilon x^{\prime}(t)=\lambda x(t), \\
x(0)=x(2 \pi), x^{\prime}(0)=x^{\prime}(2 \pi),
\end{array}\right.
$$

with associated $2 \pi$-periodic boundary conditions.

Here as $E$ we take the two-codimensional closed subspace of the functions $x \in$ $C^{2}([0,2 \pi], \mathbb{R})$ such that $x(0)=x(2 \pi)$ and $x^{\prime}(0)=x^{\prime}(2 \pi)$. As target space $F$ we consider $C^{0}([0,2 \pi], \mathbb{R})$. Since the perturbed problem is linear, without loss of generality, we may assume that $\Omega$ is unit ball of $E$. Moreover

- $L: E \rightarrow F$ is the operator $x \mapsto x^{\prime \prime}$,

- $N: E \rightarrow F$ is the (linear) map $x \mapsto x^{\prime}$,

- $C$ is the inclusion of $E$ into $F$.

Notice that $L$ is Fredholm of index zero, because it is the composition of the inclusion of $E$ into $C^{2}([0,2 \pi], \mathbb{R})$, which is Fredholm of index -2 , with the operator

$$
x \in C^{2}([0,2 \pi], \mathbb{R}) \mapsto x^{\prime \prime} \in F,
$$


which is Fredholm of index 2 (being surjective with two-dimensional kernel). The unperturbed equation $L x-\lambda C x=0$ has infinitely many eigenvalues. Namely, $0,-1,-4,-9$, $\ldots,-n^{2}, \ldots$ Here we consider $\lambda_{0}=-1$. The kernel of $L-\lambda_{0} C$ is spanned by $\sin t$ and $\cos t$. Therefore, the transversality condition (3.7) is satisfied, since no linear combination of these two elements belongs to the image of the operator $L-\lambda_{0} C$, except the trivial one (see Remark 3.1). The map $N$, which in this case is linear, is compact, because it is the composition of the compact inclusion of $E$ into $C^{1}([0,2 \pi], \mathbb{R})$ with the continuous map $x \mapsto x^{\prime}$. Obviously, the inclusion $C$ is compact as well.

One can verify that, when $\varepsilon \neq 0$ and $\lambda \neq 0$, the equation

$$
L x+\varepsilon N(x)=\lambda C x
$$

admits only the trivial solution. Therefore, the trivial eigenpair $(0,-1)$ is isolated in the set $\mathcal{E}$ of all the eigenpairs, showing that, in Theorem 3.7, the assumption that the kernel of $L-\lambda_{0} C$ is odd dimensional cannot be removed.

Actually, as one can see, any other trivial eigenpair, except $(0,0)$, is isolated. According to Theorem 3.7, the kernel of $L$ being one dimensional, $(0,0)$ must belong to an unbounded component of $\mathcal{E}$. In fact, this component is the $\varepsilon$-axis.

The following is an example of a linear system of two coupled first order differential equations showing the existence of a bounded branch of eigenpairs connecting two different trivial elements.

Example 4.5 Consider the following system of coupled differential equations with $2 \pi$ periodic boundary conditions:

$$
\left\{\begin{array}{l}
x^{\prime}(t)+x(t)-\varepsilon x(t)=\lambda y(t), \\
y^{\prime}(t)-y(t)-\varepsilon y(t)=-\lambda x(t), \\
x(0)=x(2 \pi), y(0)=y(2 \pi) .
\end{array}\right.
$$

In this example, $E$ is the two-codimensional closed subspace of the pairs of functions $(x, y) \in$ $C^{1}\left([0,2 \pi], \mathbb{R}^{2}\right)$ such that $x(0)=x(2 \pi)$ and $y(0)=y(2 \pi)$. The space $F$ is $C^{0}\left([0,2 \pi], \mathbb{R}^{2}\right)$. Since we are dealing with a linear problem, without loss of generality, we assume that $\Omega$ is the unit ball of $E$, endowed with any Banach norm among the infinitely many equivalent ones of $C^{1}\left([0,2 \pi], \mathbb{R}^{2}\right)$. For example, we may consider the following:

$$
\|z\|=\sup \{|z(t)|: t \in[0,2 \pi]\}+\sup \left\{\left|z^{\prime}(t)\right|: t \in[0,2 \pi]\right\},
$$

where $z(t)=(x(t), y(t))$, and $|\cdot|$ denotes the Euclidean norm in $\mathbb{R}^{2}$.

Notice that $E$ has codimension 2 in $C^{1}\left([0,2 \pi], \mathbb{R}^{2}\right)$ and, consequently, the operator $L: E \rightarrow F$, given by $(x, y) \mapsto\left(x^{\prime}+x, y^{\prime}-y\right)$, is Fredholm of index zero. In fact, $L$ is the restriction to $E$ of a surjective operator with two-dimensional kernel.

The operators $N$ and $C$ are defined as $(x, y) \mapsto(-x,-y)$ and $(x, y) \mapsto(y,-x)$, respectively. They are compact, because of the compact inclusion of $C^{1}\left([0,2 \pi], \mathbb{R}^{2}\right)$ into $C^{0}\left([0,2 \pi], \mathbb{R}^{2}\right)$.

Thus, our problem can be written as

$$
\left\{\begin{array}{l}
L x+\varepsilon N(x)=\lambda C x, \\
\|x\|=1,
\end{array}\right.
$$


The system of two coupled differential equations

$$
\left\{\begin{array}{l}
x^{\prime}(t)+x(t)-\varepsilon x(t)=\lambda y(t), \\
y^{\prime}(t)-y(t)-\varepsilon y(t)=-\lambda x(t)
\end{array}\right.
$$

can be represented in a matrix form as

$$
\left(\begin{array}{l}
x^{\prime}(t) \\
y^{\prime}(t)
\end{array}\right)=\left(\begin{array}{cc}
\varepsilon-1 & \lambda \\
-\lambda & \varepsilon+1
\end{array}\right)\left(\begin{array}{l}
x(t) \\
y(t)
\end{array}\right),
$$

where, given $\varepsilon$ and $\lambda$, the eigenvalues of the matrix

$$
A(\varepsilon, \lambda)=\left(\begin{array}{cc}
\varepsilon-1 & \lambda \\
-\lambda & \varepsilon+1
\end{array}\right)
$$

are $\varepsilon \pm \sqrt{1-\lambda^{2}}$. Therefore, if $|\lambda|>1$ there are nontrivial $2 \pi$-periodic solutions of (4.9) if and only if $\varepsilon=0$ and $\lambda$ is such that $\sqrt{\lambda^{2}-1} \in \mathbb{N}$, and these solutions are nonconstant. If $|\lambda| \leq 1$, the system admits nontrivial $2 \pi$-periodic solutions if and only if $\varepsilon^{2}+\lambda^{2}=1$, and these solutions are constant.

Consequently the set $\mathcal{E}$ of the eigenpairs of (4.8) is

$$
\left\{(\varepsilon, \lambda) \in \mathbb{R}^{2}: \varepsilon^{2}+\lambda^{2}=1\right\} \cup\left\{(0, \lambda) \in \mathbb{R}^{2}: \lambda= \pm \sqrt{1+n^{2}}, n \in \mathbb{N}\right\} .
$$

Observe that any trivial eigenpair $\left(0, \lambda_{0}\right)$ with $\left|\lambda_{0}\right|>1$ is isolated in the set $\mathcal{E}$. Therefore, according to Theorem 3.7, if the transversality condition (3.7) is satisfied (below we will show that it is satisfied if $\lambda_{0}=\sqrt{2}$ ), the kernel of the operator $L-\lambda_{0} C$ must be even dimensional and, thus, in this case, two dimensional.

As in the Example 4.1, the eigenpairs of the circle $\left\{(\varepsilon, \lambda) \in \mathbb{R}^{2}: \varepsilon^{2}+\lambda^{2}=1\right\}$ can be represented parametrically. In this case we set $(\varepsilon, \lambda)=(\cos \alpha, \sin \alpha)$, with $\alpha \in[0,2 \pi]$. Thus, as one can show, given any $\alpha$, the kernel of the linear operator

$$
L+(\cos \alpha) N-(\sin \alpha) C
$$

is the straight line through the origin of $E$ and the constant function

$$
z_{\alpha}:[0,2 \pi] \rightarrow E, \quad t \mapsto(\cos (\alpha / 2), \sin (\alpha / 2)),
$$

whose norm is one, $z_{\alpha}^{\prime}$ being the null function.

If we consider $\left(z_{\pi / 2}, 0,1\right)$ as a starting trivial solution of $(4.7)$, we see that even in this example the assertion of the Conjecture 3.9 is satisfied. In fact, in the space $\mathcal{S}$ of the solution triples of (4.7), the connected component containing $\left(z_{\pi / 2}, 0,1\right)$ is the image of the curve $\alpha \in[0,4 \pi] \mapsto\left(z_{\alpha}, \cos \alpha, \sin \alpha\right)$. The projection into $\mathbb{R}^{2}$ of this component, i.e., the circle $\left\{(\varepsilon, \lambda) \in \mathbb{R}^{2}: \varepsilon^{2}+\lambda^{2}=1\right\}$, is the connected component in $\mathcal{E}$ containing the trivial eigenpair $\left(0, \lambda_{0}\right)=(0,1)$ for which, as we shall see, the transversality condition is satisfied. This component, according to Theorem 3.7, contains another trivial eigenpair, which is $(0,-1)$.

We close this example by checking the transversality condition (3.7) at the eigenvalues 1 and $\sqrt{2}$ of the unperturbed operator $L-\lambda C$.

Let us consider, first, $\lambda_{0}=1$. As we have seen above, the kernel of the unperturbed operator $L-\lambda_{0} C$ (i.e., $L-C$ in this case) is the straight line through the origin of $E$ and the constant function $z_{\pi / 2}: t \mapsto(\sqrt{2} / 2, \sqrt{2} / 2)$. Let us determine the image of $L-C$. We claim that a necessary condition for a pair of functions $(u, v) \in C^{0}\left([0,2 \pi], \mathbb{R}^{2}\right)$ to belong to the image of $L-C$ is the following one:

$$
\int_{0}^{2 \pi}(u(t)-v(t)) \mathrm{d} t=0
$$


In fact, if $(u, v)$ is in the image of $L-C$, then there exists a pair of functions $(x, y) \in E$ such that $x^{\prime}+x-y=u$ and $y^{\prime}-y+x=v$, and our claim follows immediately by integrating the function

$$
u-v=\left(x^{\prime}+x-y\right)-\left(y^{\prime}-y+x\right)
$$

from 0 to $2 \pi$, and recalling the boundary conditions on $x$ and $y$. Now, observe that the necessary condition for $(u, v)$ to be in the image of $L-C$ is also sufficient, because of the fact that $L-C \in \Phi_{0}(E, F)$ and, consequently, $(L-C)(E)$ must be one codimensional in $F$ (recall that $C$ is a compact operator and $L \in \Phi_{0}(E, F)$ ). Now, once one knows both the kernel and the image of $L-\lambda_{0} C$, to investigate whether or not the unperturbed problem satisfies $\left(\right.$ at $\left.\lambda_{0}\right)$ the transversality condition (3.7) is an easy task. For example, one may utilize Remark 3.1, replacing $L$ with $L-\lambda_{0} C$ (which is still Fredholm of index zero, $C$ being a compact operator). The condition (3.7) is satisfied at $\lambda_{0}=1$.

Let us now consider the case $\lambda_{0}=\sqrt{2}$. The kernel of $L-\sqrt{2} C$ is generated by the following two linearly independent elements of $E$ :

$$
(\sqrt{2} \sin t, \cos t+\sin t), \quad(\sqrt{2} \cos t, \cos t-\sin t) .
$$

This implies, in particular, that the image of $L-\sqrt{2} C$ has codimension 2 in $F$. Therefore, it is the set of zeroes of two linearly independent functionals on $F$. Some computations show that two of these functionals are given by

$$
\phi_{1}(u, v)=\int_{0}^{2 \pi}\left(\alpha_{1}(t) u(t)+\beta_{1}(t) v(t)\right) \mathrm{d} t
$$

and

$$
\phi_{2}(u, v)=\int_{0}^{2 \pi}\left(\alpha_{2}(t) u(t)+\beta_{2}(t) v(t)\right) \mathrm{d} t
$$

where

$$
\alpha_{1}(t)=\sqrt{2} \sin t, \beta_{1}(t)=\cos t-\sin t, \alpha_{2}(t)=\sqrt{2} \cos t, \beta_{2}(t)=-\cos t-\sin t .
$$

To check the validity of our assertion, one should take into account that if the condition " $\phi_{1}(u, v)=0$ and $\phi_{2}(u, v)=0$ " is necessary for $(u, v)$ to be in the image of $L-\sqrt{2} C$, then it is also sufficient, due to the fact that if two subspaces of $F$ of the same finite codimension have the property that one is contained in the other, then they coincide.

Finally, since we know the kernel and the image of $L-\sqrt{2} C$, it is not difficult to show that even in this case the transversality condition (3.7) is satisfied. Thus, the nonexistence of a connected subset of eigenpairs containing $(0, \sqrt{2})$ and satisfying the assertion of Theorem 3.7 can be exclusively attributed to the even dimensionality of $\operatorname{Ker}(L-\sqrt{2} C)$.

We close with a very simple example showing that, in Theorem 3.7, the transversality assumption cannot be removed.

Example 4.6 Let $E=F=\mathbb{R}^{2}$, with the standard $x$ and $y$ coordinates, and consider the linear problem

$$
\left\{\begin{array}{l}
-\varepsilon y=\lambda x, \\
x+\varepsilon x=\lambda y, \\
x^{2}+y^{2}=1 .
\end{array}\right.
$$


Here, the operators $L, N$ and $C$ are, respectively, $(x, y) \mapsto(0, x),(x, y) \mapsto(-y, x)$ and $(x, y) \mapsto(x, y)$. The unperturbed equation $L x-\lambda C x$ has a unique (double) eigenvalue, $\lambda_{0}=0$, and the kernel of $L-\lambda_{0} C=L$ is one dimensional. As one can easily verify, in the set $\mathcal{E}$ of the eigenpairs of problem (4.10), the connected component containing the trivial eigenpair $(0,0)$ is the circle $\varepsilon(\varepsilon-1)+\lambda^{2}=0$. Therefore, the assertion of Theorem 3.7 is not satisfied, implying that the condition

$$
\left(L-\lambda_{0} C\right)(E)+C\left(\operatorname{Ker}\left(L-\lambda_{0} C\right)\right)=F
$$

does not hold. Consequently, in Theorem 3.7, this transversality assumption is not superfluous.

Acknowledgements We are grateful to Professor Raffaele Chiappinelli whose skillful suggestions have helped us to improve the final text of this article. We also thank the anonymous referee for his/her careful reading of the manuscript and for his/her suggestions improving the exposition of the paper.

\section{References}

1. Amann, H., Weiss, S.A.: On the uniqueness of the topological degree. Math. Z. 130, 39-54 (1973)

2. Appell, J., De Pascale, E., Vignoli, A.: Nonlinear Spectral Theory. de Gruyter, Berlin (2004)

3. Benevieri, P., Calamai, A., Furi, M.: A degree theory for a class of perturbed Fredholm maps. Fixed Point Theory Appl. 2, 185-206 (2005)

4. Benevieri, P., Calamai, A., Furi, M.: A degree theory for a class of perturbed Fredholm maps II. Fixed Point Theory Appl. 1, 20 (2006). (Art. ID 27154)

5. Benevieri, P., Calamai, A., Furi, M.: On the degree for oriented quasi-Fredholm maps: its uniqueness and its effective extension of the Leray-Schauder degree. Topol. Methods Nonlinear Anal. 46, 401-430 (2015)

6. Benevieri, P., Calamai, A., Furi, M., Pera, M.P.: On the persistence of the eigenvalues of a perturbed Fredholm operator of index zero under nonsmooth perturbations. Z. Anal. Anwend. 36, 99-128 (2017)

7. Chiappinelli, R.: Isolated connected eigenvalues in nonlinear spectral theory. Nonlinear Funct. Anal. Appl. 8, 557-579 (2003)

8. Chiappinelli, R., Furi, M., Pera, M.P.: Normalized eigenvectors of a perturbed linear operator via general bifurcation. Glasg. Math. J. 50, 303-318 (2008)

9. Chiappinelli, R., Furi, M., Pera, M.P.: Topological persistence of the normalized eigenvectors of a perturbed self-adjoint operator. Appl. Math. Lett. 23, 193-197 (2010)

10. Chiappinelli, R., Furi, M., Pera, M.P.: Persistence of the normalized eigenvectors of a perturbed operator in the variational case. Glasg. Math. J. 55(3), 629-638 (2013)

11. Chiappinelli, R., Furi, M., Pera, M.P.: Topological persistence of the unit eigenvectors of a perturbed Fredholm operator of index zero. Z. Anal. Anwend. 33, 347-367 (2014)

12. Dugundji, J.: An extension of Tietze's theorem. Pac. J. Math. 3, 353-367 (1951)

13. Furi, M., Pera, M.P.: A continuation principle for periodic solutions of forced motion equations on manifolds and applications to bifurcation theory. Pac. J. Math. 160, 219-244 (1993)

14. Guillemin, V., Pollack, A.: Differential Topology. Prentice-Hall Inc., Englewood Cliffs (1974)

15. Leray, J., Schauder, J.: Topologie et équations fonctionnelles. Ann. Sci. École Norm. Supér. 51(3), 45-78 (1934). (French)

16. Kuratowski, C.: Topologie. Vol. I. (French) 4ème éd. Monografie Matematyczne, Tom 20. Państwowe Wydawnictwo Naukowe, Warsaw. xiii+494 pp. (1958)

17. Milnor, J.W.: Topology from the Differentiable Viewpoint. University Press of Virginia, Charlottesville (1965)

18. Väth, M.: Topological Analysis: From the Basics to the Triple Degree for Nonlinear Fredholm Inclusions. de Gruyter, Berlin (2012) 